\title{
Intermediate Guardianship Based Routing Protocol In MANET
}

\author{
Arati Patel ${ }^{1}$, Rajan Patel ${ }^{2}$ \\ ${ }^{12}$ (Department of Computer Engineering, Sankalchand Patel College of Engineering, Visnagar, India)
}

\begin{abstract}
Mobile Ad hoc network (MANET) is wireless infrastructure-free network. Nodes are connected via wireless channels and can use multiple hops to exchange data. MANETs are characterized by large end to end communication latency and lack of end to end path from a source node to its destination node. Energy is indispensable factor in case of Ad hoc networks. Routing in such networks is a challenge because of characteristics. We proposed to improve the performance of AODV as intermediate hop. This intermediate hop takes custody of message when energy of other nodes is low or in the case of link failure. It is intermediate hop's responsibility to forward this message to destination. During flooding of RREQ, the node only receives RREQ if there is sufficient energy compare to threshold value. This mechanism is used to maintain the energy consumption balanced among all participating nodes. In this Approach Intermediate nodes behaves as guardian of that message leads to Performance results close to best case.
\end{abstract}

Keywords: AODV, Energy Consumption, Routing

\section{Introduction}

In recent years, mobile computing has acquired a remarkable rise in popularity which has largely been facilitated by the improvements in technology and computing power of the devices. A mobile Ad hoc network is a collection of mobile nodes that communicate to each-other over relatively bandwidth constrained wireless links [1]. The IEEE 802.11 subcommittee defines an Ad hoc network as a wireless network composed of stations within mutual communication range of each other, created in a spontaneous manner and is limited only by temporal and spatial constraints [2]. They have the advantages of rapid deployment, robustness, flexibility and inherent support for mobility [2].

In MANETs, mobile nodes equipped with the transmitter and receiver, connected wirelessly without any existing infrastructure. This type of network is defined with characteristics such as purpose-specific, dynamic and autonomous [3]. Devices are connected through distributed system in fixed wireless network while in Ad hoc network, mobile nodes are connected through decentralize manner. Node can use multiple hops to communicate with other nodes. Nodes rely on each other, so that each node acts as a router for forwarding and receiving messages to/from other nodes. Therefore in mobile Ad hoc network, a message can transfer from source to destination either with the help of intermediate nodes, or directly. Mobile Ad hoc network routing protocols classified in three parts: Reactive or on demand routing protocols, Proactive or tables driven routing protocols and Hybrid [4].

Routing in Ad hoc networks is different from traditional fixed routing protocols, since most of the existing internet protocols, whose were designed to support routing with fixed structure in a network while Ad hoc network's routing protocols have to be capable of dealing with the dynamic nature of these networks. Several routing schemes have been proposed to deal with routings in MANETs. For example, in a military application a low probability of detection and interception is a key factor, while in a sensor application minimum energy usage is more important [5]. Some of them algorithms use unicast and others use broadcasting message. One of the popular reactive routing protocol is Ad hoc On-demand Distance Vector (AODV) routing protocol, which is capable of both multicast and unicast Routing [6].

We propose an intermediate guardianship based routing protocol, which is the extended version of AODV routing protocol. In intermittent connected ad hoc networks, the destination is not always reachable by the source node. In our protocol, source needs intermediate nodes to relay messages to the destination. With the use of this message passing strategy, the probability of reaching to the destination is high.

The rest of the paper is organized as follows, Section 2 discusses background theory of MANET. Section 3 discusses some existing approaches and related work in the area. Section 4 describes the intermediate guardianship based routing protocol and Section 5 presents some concluding remarks and discusses the plan of future work.

\section{Background Theory}

Ad hoc networks are based on the assumption that all nodes collaborate to realize network services. These Networks are said to be future networks and have been receiving attention during the last few years. This 
popularity of MANET is because of wide range of available wireless services providing ubiquitous computing at low cost. AODV Routing Protocol in MANET, is the on demand type Protocol.

The Ad hoc On-Demand Distance Vector Routing Protocol (AODV) [4] is a routing protocol classified under the category of reactive protocol of MANETs. AODV is capable of both multicast and unicast routing.

\subsection{Working of AODV}

In AODV routing algorithm have mainly three control messages: Route Requests (RREQs), Route Replies (RREPs), and Route Errors (RERRs). AODV Routing protocol is working in two phases: Route Discovery and Route Maintenance.

\section{Route Discovery}

In the network, a source node needs a path to destination node, But In the routing table, there is not valid information. To find the Route of destination node, the source node broadcasts a route request (RREQ) in network. When each node receives the RREQ, it creates or updates a reverse route to the source node in the routing table and maintains it until message reaches to destination or path failure message receives. In the routing table, if it does not have a valid path to the destination node. It rebroadcasts the RREQ to neighbor nodes. When RREQ flooding from the source node to destination node, the destination node creates the reverse route, unicast route reply (RREP).

For example, Fig. 1 shows the process of the route discovery, in which the source node $\mathrm{S}$ broadcasts the RREQ and Fig. 2 shows that the destination node D unicast the RREP. If any intermediate node has the valid route to the destination node in their routing table when it receives the RREQ, then it unicast the RREP to the source node instead of the destination node. During the route discovery, when each node receives the RREQ that it has already processed, it discards the RREQ, so the loop is avoided and the overhead becomes low.

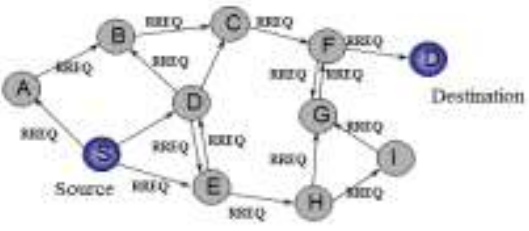

Fig. 1. Mobile nodes in MANETs broadcast RREQ

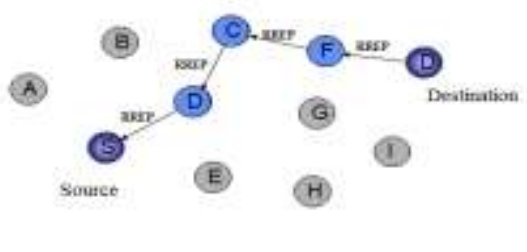

Fig. 2. Mobile nodes in MANETs unicast RREP

\section{Route Maintenance}

Each node broadcast a Hello message periodically to ensure the local connectivity. It broadcast the RREP with TTL value 1 as the Hello message. When the node does not receive any control packets from a neighbor node during few seconds, it assumes that link break to the neighbor node. In addition, when the node has the link break to the neighbor, such failures could be detected by using link layer acknowledgments. When the node that detects the link break is close to the destination node, it requires a new route to the destination node, which is known as Local Repair.

When the local repair is unsuccessful, or when the link break is far from the destination node, the node propagates a route error packet (RERR) to the source node.

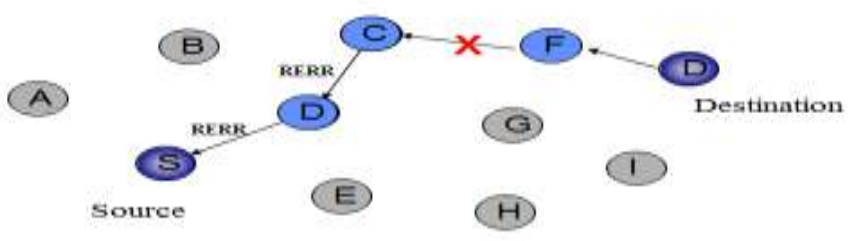

Fig. 3. Intermediate node propagates RERR message

\section{Related Work}

MANETs are partitioned wireless Ad hoc networks with intermittent connectivity. Nodes rarely have information on the changing network topology in an intermittently connected network or a mobile network. 
Nodes may not know the availability of future connection, but the network may benefit from routing entry of nodes contact over time.

There are many approaches proposed for routing in MANETs. One of the popular routing protocol is the AODV [6]. AODV Routing protocol is reactive type protocol and is working in two phases, Route Discovery and Route Maintenance. In AODV routing algorithm have mainly three control messages: Route Requests (RREQs), Route Errors (RERRs), and Route Replies (RREPs). When a source node needs a path to destination node and there is not the valid route information in the routing table, the source node broadcasts a route request packet (RREQ) in the network for find route of destination node. When each node receives the RREQ, it creates or updates a reverse route to the source node in the routing table and maintains it until message reaches to destination or path failure message receives. Source node checks the sequence number, updates the forward route and starts communications.

Another popular traditional protocol is Dynamic Source Routing (DSR), which used the source routing instead of depending on the routing table at each intermediate device [7]. It is analogous to AODV because it creates a route on-demand when a transmitting node requests one. In Ad hoc networks, DSR is an ondemand protocol presented to control the bandwidth consumed by control packets by eliminating the periodic table-update messages mandatory in the table-driven approach. The weakness of this protocol is that the route maintenance strategy does not locally repair a broken link. The connection configuration delay is higher than in table-driven approach.

In multi-hop AODV algorithm, author implemented new approach, AODV-2T [8], By applying a conservative and circumspect concept, an improved AODV designed to compromise link failures problems. Before Link Breaking, AODV-2T could beat the problem by preparing a backup route just-in-time. AODV-2T provides route maintenance strategy, how to find a backup route. Thus, if we improve backup route establishing function of AODV-2T to support Multi-hop backup route, the improved protocol should enhance the Ad hoc networking performance both in term of number of route break decrement which in turn increases the network's throughput.

AODV-2T which is improves the throughput of Ad hoc network and reducing the broken route to applying 2 level threshold concept. AODV-2T is gives better performance compared to original AODV. Another energy efficient LEA-AODV routing protocol [9] is simple but efficient algorithm which is balance energy consumption among all participating nodes. That will extending the network lifetime of the batteries that power these MANET based on one of the most important routing protocols AODV. Nodes don't have the appropriate quantity of energy to be used in routing process that LEA-AODV is difficult to take off. The established routes will rely on the local decision of each node.

\section{Proposed Approach}

In the discontinuous mobile Ad hoc network, there is no direct path available between all source and destination and nodes are moving so link failure during transmission is happened frequently. The proposed algorithm is described in two phases. The first phase of the algorithm concentrates on energy efficient routing. We referenced LEA-AODV protocol [9] to minimize the power consumption of nodes. In LEA-AODV algorithm, the author has used mechanism of balancing the energy consumption among all participating nodes. Due to dynamic nature of mobile network, nodes are free to move at any time so the maintenance of routing path during message transmission is very difficult, and it may lead to link failure. We are focusing this problem by giving the responsibility of message to the intermediate node and it is covered in another phase of algorithm.

When node wants to send message and destination is not in range then it starts the broadcasting of RREQ control message. The neighbour nodes receive RREQ and compare its current battery level with the threshold $(\theta)$. Threshold value is dynamically changing and will be derived based on the average power of participated nodes along the path. Using the following formula, which is referred from LEA_AODV [6], nodes calculate the threshold

$$
\theta=\frac{\sum_{i=1}^{n} n \_ \text {power }}{n}
$$

Where $\mathrm{n}$ is the number of nodes inside current node region, $n \_p o w e r i$ is the power level of each node link. If node's current energy is higher than threshold, it will respond to the RREQ as usual. Otherwise, it will simply drop it. This technique leads the network to ensure that all the nodes are balanced in their energy consumption.

In the another phase of this proposed algorithm, we extended AODV algorithm as intermediate nodes become guardian, which is useful in the case of link failure between two nodes. Source node is broadcasting RREQ and intermediate nodes receive it and continue this process until RREQ reaches to destination node. After receiving RREQ message, destination forwards RREP message to the source through the same path with the help of intermediate nodes. Finally source sends message when it receives RREP from destination. Any 
node from routing path may be disconnected with his neighbour node and link failure occurs between them. In this situation, node closest to source, who participated in link failure, sends RERR to inform about link error to source in traditional AODV. We modified this procedure as in the situation of link failure, intermediate node takes responsibility of coming data message instead of sending RERR message to source node. And the intermediate node takes custody of message. Now this node pretends as source node and broadcasts RREQ to find path of destination node. In most of the scenario, this guardianship based approach optimizes the routing and reduces the overhead of network.

We consider an example to explain this proposed solution. Suppose MANET consist of seven mobile nodes, which is shown in below figures.

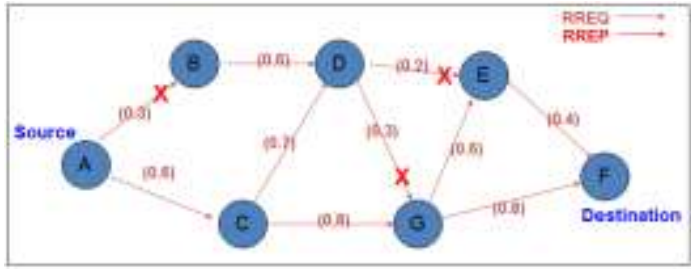

Fig. 4. Nodes broadcast RREQ

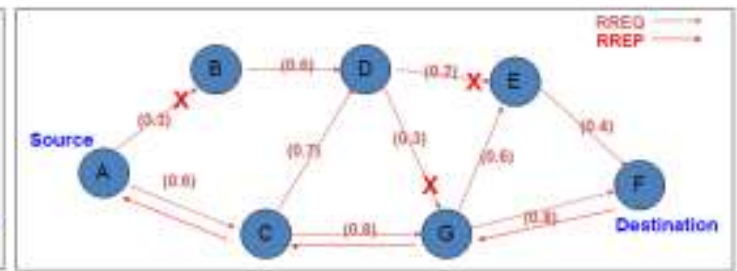

Fig. 5. Nodes unicast RREP

Source node A has message $\mathrm{M}$ which destination is node $\mathrm{F}$. Based on the scenario, we assume the initial value of the threshold is 0.5 , it can be calculated as the average ratio of remaining energy of each link node. If $\mathrm{A}$ is connected by the direct edge $\mathrm{B}$ and $\mathrm{C}$, here $\mathrm{A} \rightarrow \mathrm{B}$ is 0.3 and $\mathrm{A} \rightarrow \mathrm{C}$ is 0.6 , the $\theta>0.3$ so RREQ is simply dropped by node $\mathrm{B}$ and the request path is passing through node $\mathrm{C}$. This procedure will be executed up to RREQ is received by destination.

When destination received RREQ, it sends back route reply to inform source about routing path. Algorithm chooses the best routing path in terms of hop count for sending message, here A $\rightarrow \mathrm{C} \rightarrow \mathrm{G} \rightarrow \mathrm{F}$ is optimal path because of fewer hop count so destination sends RREP through this path. link failure between $\mathrm{G}-$ $F, G$ sends RERR message to A.

In proposed algorithm, we modify route error message procedure and don't send RERR to source and last node of that available path takes custody of message. In this scenario, node G takes responsibility of message M. Now node G broadcasts RREQ message to find destination node of message M. Hypothetically it's reduce the overhead and delay of network.

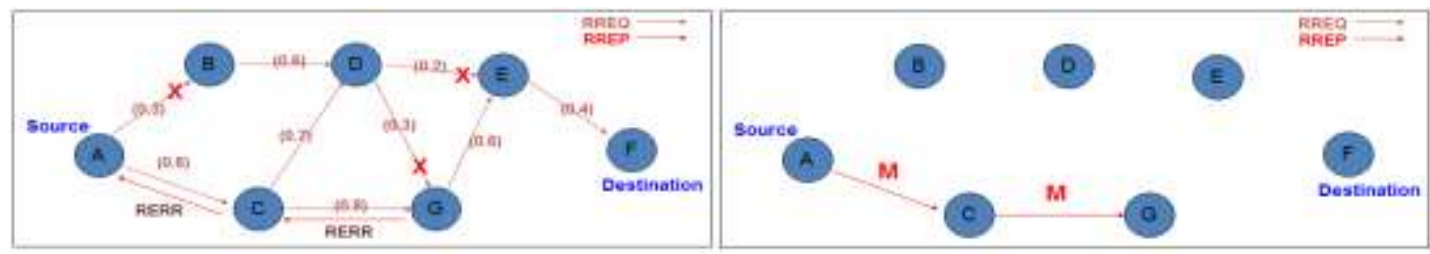

Fig. 6. Nodes forwards RERR

Fig. 7. Source Node sends data message to guardian node

The Fig. 8. Intermediate Guardianship approach is work on this below Algorithm

\section{Algorithm}

Step 1. Source node, which has message to send, broadcasts RREQ in the network to find route of destination Node.

Step 2. When there is an interaction between two nodes $\mathrm{n} 1$ and $\mathrm{n} 2$, $\mathrm{n} 1$ sends RREQ to $\mathrm{n} 2$.

Step 3. After receiving RREQ, node $\mathrm{n} 2$ will calculate the threshold value $\theta$ as

$$
\theta=\frac{\sum_{i=1}^{n} n_{\text {power }}}{n}
$$

Where,

$$
\begin{aligned}
& \mathrm{n}=\text { number of nodes around the backward path, } \\
& n \_ \text {power } i=\text { power level of each node link. }
\end{aligned}
$$

Step 4. If energy of node $\mathrm{n} 2>\theta$, then node $\mathrm{n} 2$ will make an entry in the routing table and will broadcast it further.

Step 5. Otherwise node $\mathrm{n} 2$ drops RREQ.

Step 6. Continue these steps [2-4] until RREQ reaches to the destination.

Step 7. Destination node will send RREP to source when RREQ has reached the destination node and source 
forwards data through this route.

Step 8. During data transmission, if any link of route is disconnected, then instead of sending RERR, intermediate nodes receives data message and become guardian. Now this guardian node broadcasts RREQ to find remaining path and follow these steps until message reaches to destination

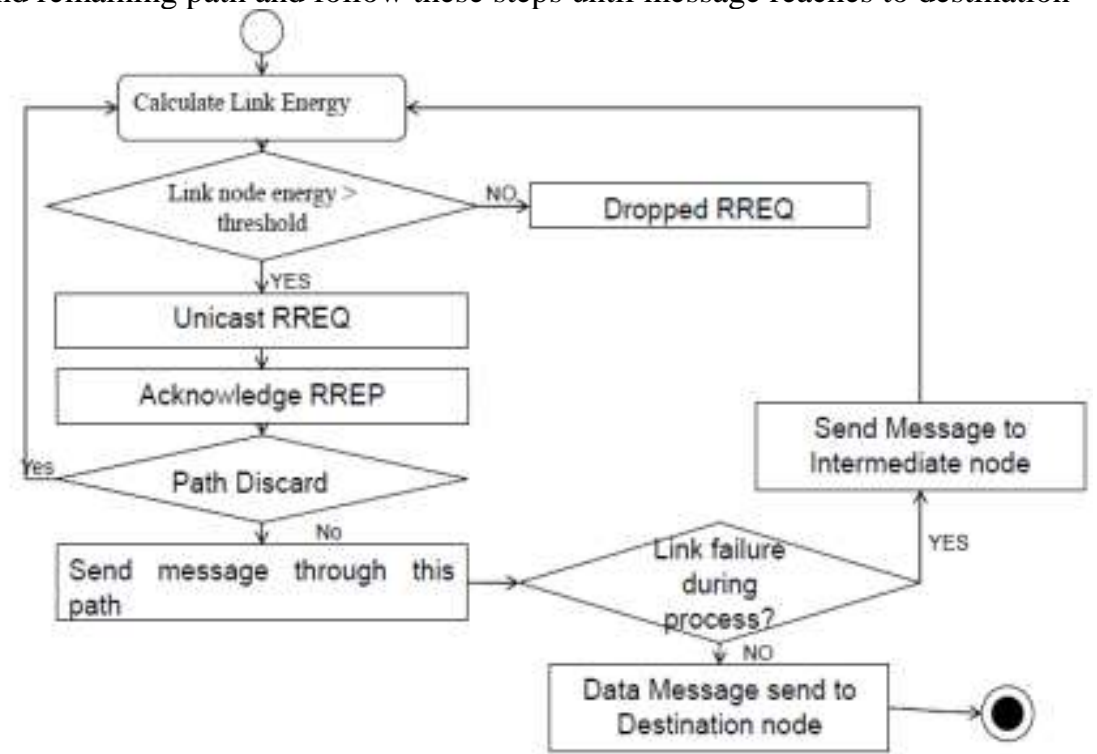

Fig. 8. Intermediate Guardianship approch

\section{Conclusion}

Detail study of routing protocols show that there are many approach to use intermediate node and its behavior for routing decision. In Ad hoc network, for efficiency and reducing overhead of network, routing decision has been a focal point of research in this area. As discuss in propose solution, we try to improve the performance by enhancing LEA-AODV routing protocol by applying modified procedure in the circumstance of link failure and using energy threshold for remain energy when battery's level is critical. We are planning to implement propose routing strategy in NS-2 simulator. Take results and do performance evolution and analysis and make conclusion of policy.

\section{References}

[1]. Toh,C.-K., Delwar, M., Allen,D.: Evaluating the communication performance of an ad hoc wireless network. Wireless Communications, IEEE Transactions on , vol.1, no.3, 2002, pp. 402-414

[2]. IEEE Standard for Information Technology- Telecommunications and Information Exchange Between Systems-Local and Metropolitan Area Networks-Specific Requirements-Part 11: Wireless LAN Medium Access Control (MAC) and Physical Layer (PHY) Specifications, IEEE 1997

[3]. Lidong, Z. ,Zygmunt, J.H. :Securing Ad Hoc Networks, Network, IEEE Volume 13, Issue 6, 1999, pp, 24-30

[4]. Uma Rathore Bhatt, Priyanka Jain, RakshaUpadhyay.: Enhanced AODV- An Energy Efficient Routing Protocol for MANET, IEEE 2013

[5]. Kuosmanen, P. Classification of Ad Hoc routing protocols. Finnish Defence Forces, Naval Academy, Finland, petteri.kuosmanen@ mil.fi 2002

[6]. C. Perkins, E. Belding Royer, S. Das :Ad hoc on-demand Distance Vector (AODV) Routing, IETF RFC3561,2003

[7]. Johnson DB, Maltz DA. Dynamic source routing in ad hoc wireless networks. In: Mobile computing: Springer; 1996

[8]. Akkaphop, O., Mayuree, L.:An Improvement of Ad Hoc NetworkRoute Maintenance, Proceeding of International Symposium on Communication and Information Technologies, (Vientiane, LaoPDR, October 2008), 102-105

[9]. AL-GabriMalek, Chunlin LI, Zhiyong Yang, Naji Hasan.: Improved the Energy of Ad Hoc On-Demand Distance Vector Routing Protocol, Science Direct, IERI Procedia 2, pp:355-361,2012 